\title{
Discours
}

Revue de linguistique, psycholinguistique et

informatique. A journal of linguistics, psycholinguistics

and computational linguistics

$1 \mid 2007$

Varia

\section{Analyse thématique automatique fondée sur la notion d'univers de discours}

Frédérik Bilhaut

\section{OpenEdition}

Journals

Édition électronique

URL : http://journals.openedition.org/discours/101

DOI : 10.4000/discours.101

ISSN : 1963-1723

Éditeur :

Laboratoire LATTICE, Presses universitaires de Caen

\section{Référence électronique}

Frédérik Bilhaut, " Analyse thématique automatique fondée sur la notion d'univers de discours », Discours [En ligne], 1 | 2007, mis en ligne le 03 mai 2008, consulté le 19 avril 2019. URL : http:// journals.openedition.org/discours/101 ; DOI : 10.4000/discours.101

Ce document a été généré automatiquement le 19 avril 2019.

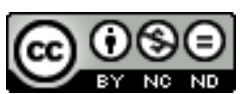

Discours est mis à disposition selon les termes de la licence Creative Commons Attribution - Pas d'Utilisation Commerciale - Pas de Modification 4.0 International. 


\title{
Analyse thématique automatique fondée sur la notion d'univers de discours
}

\author{
Frédérik Bilhaut
}

\section{Introduction}

1 Dans le champ du Traitement Automatique des Langues (TAL), le terme d'analyse thématique recouvre des réalités très diverses, tant par les objectifs poursuivis que par les méthodes mises en oeuvre. Cette diversité s'explique en partie par les difficultés rencontrées, toutes disciplines confondues, à circonscrire l'objet «thème » pour en proposer une définition théorique susceptible de guider l'analyse automatique. On observe d'ailleurs une forte inter-détermination, dans cette branche du TAL, entre les procédés développés et les objectifs visés, à tel point qu'il est parfois difficile de distinguer ce qui relève de la caractérisation de la tâche elle-même de ce qui relève de la méthode proposée pour la réaliser.

2 Ainsi les procédés fondés sur la notion de cohésion lexicale (cf. Ferret, Grau, 2001, par exemple), s'appuient-ils généralement sur une notion de segment thématique qui n'est formellement définie que par la méthode employée pour les reconnaître, et non par une quelconque définition a priori. La tâche proposée pour la récente campagne DEFT 2006 illustre parfaitement ce phénomène: il était demandé aux participants de délimiter automatiquement des segments dits "thématiques» très hétérogènes selon les constituants du corpus (paragraphes dans un discours politique, textes de lois dans un recueil, ou sections titrées dans un ouvrage scientifique). On voit mal dans ce contexte quelle caractérisation linguistique a priori pourrait unifier des segments aussi disparates.

3 L'approche que nous décrivons dans cet article s'applique à fonder un procédé d'analyse automatique sur la caractérisation préalable de ce qui constituerait un segment thématique, en s'appuyant sur des modèles d'ordre linguistique. Nous nous rendons ainsi capable de capter certains phénomènes discursifs inaccessibles aux méthodes 
" alinguistiques ", de décrire finement le contenu informationnel des segments identifiés, pour finalement produire des annotations sémantiques trouvant différentes applications dans le domaine de l'accès assisté à l'information.

Si cette voie a déjà fait l'objet de récents travaux (voir par exemple (Hernandez, 2004) ou (Lucas, Giguet, 2005)), elle reste encore faiblement représentée dans la littérature. Elle présente en effet, au moins à l'heure actuelle, l'inconvénient majeur d'être limitée à des phénomènes de langue bien particuliers, et implique donc souvent d'abandonner pour un temps les objectifs applicatifs nécessitant une couverture globale des documents et l'applicabilité à du texte tout-venant. La démarche nous paraît cependant pertinente dans la mesure où la caractérisation a priori des objets linguistiques à analyser permet de raisonner sur leurs possibles interactions avec d'autres modèles et, à terme, de les intégrer au sein d'un cadre plus général.

5 La première partie de cet article nous permettra d'expliciter les liens que l'on peut tisser entre les notions de thème et d'univers de discours. Afin d'en rendre compte plus formellement, nous définirons dans une seconde partie la notion de thème composite, avant d'en observer les manifestations discursives et de décrire la méthode d'analyse automatique qu'elle sous-tend.

\section{Hypothèses}

6 Les hypothèses que nous formulons ici concernent le rapprochement entre les notions de thème discursif d'une part, et d'univers de discours d'autre part. La première est ici envisagée en termes d'à propos, c'est à dire qu'un thème de discours se voudra représentatif de « ce dont on parle » dans un certain segment textuel. Nous considérerons qu'un tel thème devra généralement être représenté par une structure complexe, agrégeant plusieurs référents du discours. Cette idée est généralement envisagée sous l'angle propositionnel, par exemple chez Van Dijk (1977), un thème de discours formant une proposition au sens prédicatif. Mais nous explorerons ici un autre mode d'agrégation entre référents thématiques, reposant sur la notion d'univers de discours ${ }^{1}$. Un univers de discours correspond, selon la définition de Martin (1983), à "l'ensemble des circonstances, souvent spécifiées sous forme d'adverbes de phrase, dans lesquelles [une] proposition peut être dite vraie ». En d'autres termes, l'univers attaché à la description d'un événement, fait ou procès, est constitué d'un ou plusieurs critères véridictionnels contraignant l'interprétation du contenu propositionnel.

7 La méthode d'analyse thématique que nous allons décrire dans cet article est ainsi fondée sur les trois hypothèses suivantes:

1. i) les univers de discours peuvent participer à l'instauration d'un thème de discours, par un mécanisme d'agglomération de référents aboutissant à la formation d'une structure complexe que l'on pourrait qualifier de « molécule thématique »;

2. ii) dans ce cas, le pouvoir organisationnel de ces univers de discours se répercute sur la structure thématique du texte ;

3. iii) ce pouvoir organisationnel peut être signalé soit par des indices formels (connecteurs ou constructions syntaxiques particulières, par exemple), soit par des relations sémantiques en rapport avec l'organisation présupposée des connaissances. 
8 L'hypothèse (i) se reflète assez immédiatement dans la propension des univers de discours à être introduits parmi les constituants initiaux de l'énoncé (selon différents degrés d'intégration syntaxique), position par ailleurs très largement associée au phénomène de topicalisation et de définition de l'à propos. Par exemple, dans un segment débutant par "Dans l'Ouest, le taux de redoublement diminue... », on reconnaîtra deux constituants potentiellement topicaux: «dans l'Ouest» (scene-setting topic chez Lambrecht, chinese-style topic définissant un framework chez Chafe, ou theme chez Dik) et « le taux de redoublement » (topic chez Lambrecht ou Dik, subject chez Chafe). Ces deux constituants se trouvent alors en situation de "concurrence topicale", et comme le préconise l'approche pragmatique de Lambrecht, le contexte doit être pris en considérations avant d'attribuer à l'un ou l'autre le statut de topique.

9 Mais dans le cas où c'est le constituant syntaxiquement intégré qui l'emporte, la question reste posée du rôle, en termes d'à propos, de l'élément détaché. Nous nous rangeons sur ce point à l'avis de Le Goffic (1994), jugeant que dans cette situation, chacun des deux constituants joue bien un rôle dans la définition de l'à propos. Nous considérons alors que le thème de l'énoncé précédent comprend aussi bien l'univers du discours que son sujet proprement dit, formant un couple du type « le redoublement - dans l'Ouest » (pour peu que le taux de redoublement soit bien le propos des énoncés du co-texte, et non pas «l'Ouest » qui constituerait alors le thème à lui seul). Nous introduirons pour cela la notion de thème composite.

10 L'hypothèse (ii) est très immédiatement motivée par le fait que ces mêmes adverbiaux, syntaxiquement non intégrés et en position préverbale, peuvent revêtir une fonction particulière au niveau discursif, dont rend compte l'hypothèse de l'encadrement du discours (Charolles, 1997). Cette dernière décrit leur capacité à «signaler que plusieurs propositions apparaissant dans le fil d'un texte entretiennent un même rapport avec un certain critère et sont de ce fait regroupables à l'intérieur d'unités [appelées] cadres ». C'est précisément cette fonction organisationnelle qui nous permet d'avancer qu'un adverbial spécifiant un univers de discours peut non seulement intervenir dans la construction de l'à propos, mais aussi intervenir très directement dans la segmentation thématique d'un texte.

11 Nous avons cependant constaté par l'observation de corpus que dans certains cas, l'instauration d'un univers de discours semble pouvoir être réalisée sans introducteur de cadre au sens strict, tout en étant associé à un phénomène thématico-organisationnel, ce qui constitue l'hypothèse (iii). Comme nous le verrons cette situation est liée à des conditions très particulières, mais elle apparaît néanmoins fréquemment en corpus. Il nous faut donc considérer différents schémas discursifs dont la fonction est d'installer un univers de discours, y compris ceux qui ne sont pas proprement cadratifs. Pour cela, nous devons distinguer clairement la fonction discursive de ses possibles réalisations en discours, fonction qui consiste ici, selon les termes utilisés plus haut à introduire une ou plusieurs « circonstances » selon lesquelles le propos d'un segment du discours doit être interprété.

12 À partir de ces trois hypothèses, nous allons montrer comment le thème de certaines constructions discursives peut être analysé et représenté sous forme de structures que nous appelons thèmes composites. Nous décrirons par la suite une implémentation informatique visant à analyser automatiquement ces structures. 


\section{La notion de thème composite}

\subsection{Définition} d'un texte ou d'un fragment de discours nécessite de prendre en compte non seulement un topique discursif, support de l'information nouvelle, mais aussi le contexte au sein duquel doit être située cette information. Pour formaliser et représenter ce phénomène, nous utiliserons la notion de thème composite, objet constitué de deux éléments: le premier, que nous appelons noyau thématique ou simplement noyau, correspond au topique discursif du segment en tant que représentant de son à propos ; le second est luimême un ensemble d'éléments que nous appelons satellites et qui définissent l'univers de discours au sein duquel se tient le noyau. Un thème composé d'un noyau $n$ et d'un ensemble de satellites $s_{i}$, '’ensemble sera noté comme suit :

$$
\mathrm{n}-\left(\mathrm{s}_{1}, \ldots, \mathrm{s}_{\mathrm{n}}\right)
$$

Voici à titre d'exemple un énoncé suivi d'une interprétation possible en termes de thème composite :

[1] Dans l'ouest, le taux de retard scolaire est en régression depuis une dizaine d'années.

«le taux de retard scolaire» - («dans l'Ouest», «depuis une dizaine d'années »)

Précisons que notre démarche n'implique aucune hypothèse sur la nature précise de ces objets conceptuels, qui peuvent présenter leur propre structure à l'intérieur d'un thème composite. Celle d'un noyau thématique, en particulier, dépendra de l'approche adoptée : il peut correspondre à un simple référent, une proposition au sens logique, un ensemble de formes de surface, etc. Ceci est indépendant du modèle que nous proposons ici, même si l'implémentation qui sera décrite plus loin procède nécessairement à ses propres choix sur ce point.

Notre objectif étant de décrire l'à propos de segments discursifs et non seulement d'énoncés isolés, il nous faudra considérer les différentes configurations discursives susceptibles de construire des thèmes composites en discours. Il existe en effet divers « outils discursifs » susceptibles de participer à l'installation progressive des différents satellites qui participent de « ce dont on parle » dans un segment. cadres de discours, dont voici un exemple :

[2] En VFR, l'évitement des autres aéronefs et des obstacles s'effectue généralement en appliquant la règle "voir et éviter ». Il s'agit de détecter assez tôt l'éventuel danger extérieur pour concevoir et exécuter une manoeuvre d'évitement. Mais il peut arriver que le regard des occupants en place avant soit orienté longuement vers les instruments. Ainsi, la surveillance extérieure devient défaillante. (Source BEA)

Le thème composite associé pourrait alors être le suivant (on notera que l'acronyme anglais «VFR» (Visual Flying Rules, toujours employé sous cette forme en français) 
s'oppose à IFR (Intrument Flying Rules), cette opposition étant fortement discriminante dans le domaine aéronautique) :

«procédures d'évitement » - (« en VFR »)

Les thèmes composites discursifs peuvent souvent être assemblés sous forme de structures arborescentes. En complément de la notation que nous venons d'introduire, qui permet de représenter les feuilles de ces structures, nous devons donc introduire une notation applicable aux autres éléments de l'arbre. Envisageons pour cela l'exemple suivant :
[3] L'explosion des effectifs scolaires
$\{\{$ Dans l'enseignement public, elle s'accélère en Île-de-France, en Picardie, dans le Centre, ainsi qu'en Provence ; elle reste modérée dans l'Ouest et le Nord. [...] $\}_{\mathrm{s} 1}$ \{L'enseignement privé\} enregistre des baisses d'effectifs \dotuline\{en Bretagne\}, où il est fortement implanté, ainsi que dans les académies de la diagonale Pyrénées-Lorraine, où son audience est par contre traditionnellement réduite $\left.[. .].\}_{\mathrm{s} 2}\right\}_{\mathrm{s} 0}$ (Source HER)

Dans ce cas, puisque les thèmes composites de $S_{1}$ et $S_{2}$ sont caractérisés par un noyau «effectifs scolaires» et deux satellites relatifs au statut des établissements considérés ("privés » ou «publics »), nous représenterons le thème du segment englobant $S_{0}$ en utilisant la notation suivante :

$$
\mathrm{T}\left(\mathrm{s}_{0}\right)=\text { « effectifs scolaires } »-(<<\text { statut }>>)
$$

21 Cette notation nous permet de spécifier que le noyau thématique de $S_{0}$ est décrit en relation avec plusieurs entités d'un "axe sémantique " (forme de classe sémantique sur laquelle nous reviendrons par la suite) ici nommé «statut ». De la même façon, le thème composite de $S_{1}$ pourrait être noté comme suit :

$$
\mathrm{T}\left(\mathrm{S}_{1}\right)=\text { « effectifs scolaires } »-(\text { ( public } »,<<\text { spatial }>)
$$

\subsection{Caractère contextuel des thèmes composites}

Considérons l'exemple suivant :

[4]At eight o'clock this morning ${ }_{(\mathrm{P} 1)}$, the President ${ }_{(\mathrm{P} 2)}$ left from Barajas to attend the international conference to be held in Rome.

Nous avons mentionné plus haut différents concepts conduisant à distinguer dans cette phrase deux constituants potentiellement topicaux, ici marqués $\mathrm{P}_{1}$ et $\mathrm{P}_{2}$. Si l'on s'autorise à mettre en parallèle les dénominations proposées par les différents auteurs, le premier pourra être appelé "scene-setting topic", et le second "sujet» ou simplement « topique ». Conformément à ce que nous avions alors annoncé, nous considérerons ici les référents associés comme deux composantes d'un même thème composite de noyau «le président » et de satellite temporel « ce matin à huit heures ».

Ce choix est tout d'abord justifié par le fait que la notion de thème relève pour nous directement de l'à propos, et que l'on peut effectivement considérer que cette phrase concerne «ce qu'a fait le Président ce matin à huit heures». Mais il est d'autre part justifié par le fait que nous prenons en considération le discours dans son ensemble et 
non pas des phrases isolées. Nous adoptons ainsi une approche pragmatique considérant que le rôle topical d'un constituant ne peut être déterminé sans tenir compte du contexte, ou tout du moins que la structure propre à une phrase donnée ne donne que des indications "par défaut ». Il nous paraît opportun de reproduire à ce sujet ces propos de M. Charolles :

Il n'est pas possible de statuer sur le topique (aboutness) d'une phrase isolée. Par défaut de contexte ultérieur, les adverbiaux détachés en tête de phrase, n'indiquent pas ce à propos de quoi est la phrase. Mais rien n'empêche que la suite oblige à leur restituer ce statut. Ce constat, s'il est bien fondé, milite en faveur d'approches dynamiques des phénomènes de topicalisation et conduit à réintégrer dans la discussion de ces phénomènes la notion de topique de discours. (Charolles, 2003 : 47)

Pour ces raisons, nous décrirons dorénavant toujours les thèmes composites d'un segment relativement au contexte considéré: nous considérerons le thème composite d'un segment donné non pas comme une donnée absolue, mais relative à un autre segment englobant que nous dirons «de référence ». Selon le segment de référence choisi, on pourra ainsi obtenir des thèmes différents pour un même segment ${ }^{2}$. Pour les distinguer, nous noterons quand cela est nécessaire $T\left(\mathrm{~S}_{1}, \mathrm{~S}_{0}\right)$ le thème composite d'un segment $\mathrm{S}_{1}$ considéré relativement à un autre segment $\mathrm{S}_{0}$. Pour illustrer ce point, considérons les trois exemples suivants :

[5a] $\left\{\{\text { Dans le primaire, le taux de retard scolaire a fortement diminué. }\}_{\mathrm{U} 1}\right.$ Cette baisse est en partie attribuable à $[. .].\}_{\mathrm{s} 1}$

$[5 b]\left\{\{\text { Dans le primaire, le taux de retard scolaire a fortement diminué. }\}_{\mathrm{U} 2}\right.$ Cette section du système scolaire est alors celle qui $[. .].\}_{\mathrm{s} 2}$

[5c] \{ \{L'enseignement primaire a connu une forte diminution du taux de retard scolaire. $\}_{\mathrm{U} 3}$ Cette baisse est en partie attribuable à $\left.[. .].\right\}_{\mathrm{s} 3}$

Dans le premier cas, nous considérerons que le thème de la première phrase considérée isolément est $\mathrm{T}\left(\mathrm{U}_{1}, \mathrm{U}_{1}\right)=$ " baisse du retard scolaire» («dans le primaire »). La phrase suivante confirme ici ce choix "par défaut", et le thème composite de cette phrase considérée relativement à l'ensemble du segment est identique : $\mathrm{T}\left(\mathrm{U}_{1}, \mathrm{~S}_{1}\right)=\mathrm{T}\left(\mathrm{U}_{1}, \mathrm{U}_{1}\right)$. Dans le second cas, la première phrase est identique mais la seconde vient remettre en cause les choix faits a priori. Ou plutôt, le thème de $\mathrm{U}_{2}$ considérée relativement à $\mathrm{S}_{2}$ est différent du thème de cette même phrase considérée isolément, puisque $T\left(U_{2}, S_{2}\right)=$ « enseignement primaire ».Dans le troisième cas enfin, la situation est inverse, puisque $T\left(\mathrm{U}_{3}, \mathrm{U}_{3}\right)=$ « enseignement primaire » alors que $\mathrm{T}\left(\mathrm{U}_{3}, \mathrm{~S}_{3}\right)=$ « retard scolaire » - (« dans le primaire ») exactement comme dans le premier cas.

Nous reviendrons plus loin sur les configurations discursives faisant intervenir la dimension contextuelle des thèmes composites. Mais pour l'heure, nous allons considérer un certain nombre de configurations discursives moins problématiques.

\subsection{Réalisations discursives liées au détachement syntaxique}

Un exemple de thème composite discursif peut être observé dans le passage suivant :

[6] $\{\{$ Dans l'enseignement primaire $(\mathrm{P} 1)$, on assiste à une forte diminution du taux de retard scolaire dans les années 80.$\}_{\mathrm{U} 1}$ Cette baisse est en partie attribuable à la réduction du nombre d'élèves par classe, qui $[. .].\}_{\mathrm{S} 1}\{$ Dans le 
secondaire $_{(\mathrm{P} 2)}$, on assiste au contraire à une augmentation sensible du taux de retard. Celle-ci est principalement imputable à $[. .].\}_{\text {so }}$ (Construit) des thèmes discursifs. Au sein de la typologie établie par M. Charolles, les cadres qui nous intéresseront particulièrement sont dits "véridictionnels", et correspondent plus particulièrement aux cadres temporels, spatiaux, représentationnels, ou encore praxéologiques. Mais les cadres dits «thématiques» (introduits par des constructions de type « concernant $\mathrm{X} »)$, peuvent également jouer un rôle comparable dans certains cas, comme on peut l'observer dans l'exemple suivant :

[7] Le Conseil de sécurité des Nations Unies a créé en 1991 une Commission spéciale (UNSCOM) en charge du désarmement de l'Irak, tout en confiant le volet nucléaire à l'AIEA. [...] Huit années plus tard, quel bilan peut-on dresser ? [...] \{ En ce qui concerne le nucléaire ${ }_{(\mathrm{P} 1)}$, l'AIEA estime avoir épuisé dès 1995 sa mission de destruction et d'enlèvement des matières prohibées $[. .].\}_{\mathrm{S} 1}\{$ Dans le domaine biologique ${ }_{(\mathrm{P} 2)}$, l'UNSCOM a procédé à l'élimination de $\left.[. .].\right\}_{\mathrm{P} 2}$ (Source internet)

31 Cet exemple illustre la fonction thématique de satellite qui peut parfois être attribuée à un introducteur thématique : dans ce cas, cette construction participe à l'élaboration du thème composite $\mathrm{T}\left(\mathrm{S}_{1}\right)$ = "le désarmement de l'Irak» - («nucléaire »). On notera pourtant que les cadres thématiques ne sont pas considérés comme véridictionnels. Il est de fait très clair que le statut de satellite ne peut pas toujours être attribué à ce type d'introducteurs : dans certains cas, et tout particulièrement en langue peu soutenue, ils peuvent effectivement être explicitement repris, et ainsi introduire un noyau thématique. Par exemple :

[8] En ce qui concerne le nucléaire, il pollue aussi mais de façon différente [... ]

Mais il apparait cependant que dans de nombreux autres cas, cette construction est utilisée pour attribuer le statut de satellites à des expressions qui se prêtent plus difficilement à une construction adverbiale (comme "dans le nucléaire » ou «en nucléaire »). Nous serons donc amené à considérer certains introducteurs du type " concernant $\mathrm{X}$ » comme véridictionnels et non thématiques, c'est-à-dire comme définissant un satellite thématique et non un noyau. Dans d'autres cas en revanche, une reprise explicite nous invitera à les considérer comme introduisant un cadre proprement « thématique », et donc comme introduisant un noyau.

Terminons, pour ce qui est des cadres de discours, avec la possibilité de trouver des configurations caractéristiques d'un domaine, non pas seulement au niveau des concepts 
évoqués mais bien au niveau de la construction elle-même. C'est par exemple le cas dans l'extrait suivant:

[9] Chez les patientes à risque hémorragique, l'administration intraveineuse d'ocytociques avant ou après la sortie du placenta n'a pas réduit l'incidence des hémorragies de la délivrance ni la durée de la délivrance du placenta. [...] (Source APH)

"administration ... d'ocytociques» - («chez les patientes à risque hémorragique »)

Même s'ils revêtent une importance capitale dans notre approche, nous considérons les cadres de discours comme une configuration parmi d'autres relativement au problème des thèmes composites, et nous considérons qu'il existe une variété de configurations susceptibles de produire des phénomènes équivalents. L'extrait suivant fait par exemple intervenir une construction clivée :

[10] \{ En théorie, le district de Lüchow-Dannenberg dispose dans tous les domaines d'un potentiel suffisant pour couvrir la totalité de son approvisionnement énergétique à partir de ressources renouvelables. \{C'est dans le secteur de l'électricité ${ }_{(\mathrm{P})}$ que cet objectif est le plus facile à réaliser. [...]\} ${ }_{S 1}\left\{\right.$ Un inconvénient pour le secteur thermique $e_{(\mathrm{P} 2)}$ tient au fait que le district ne dispose de pratiquement aucun réseau de chauffage urbain. $\}_{\mathrm{S} 2}$ \{Les gros clients qui seraient à même de rentabiliser l'exploitation de la géothermie $e_{(\mathrm{P} 3)}$ font eux aussi défaut. $\left.\}_{\mathrm{s} 3}\right\}_{\mathrm{s} 0}$ (Source EC)

Voici l'analyse en thème composite que l'on pourrait donner pour ce passage, en notant $E_{0}$ le noyau thématique «l'approvisionnement énergétique du district de LüchowDannenberg »:

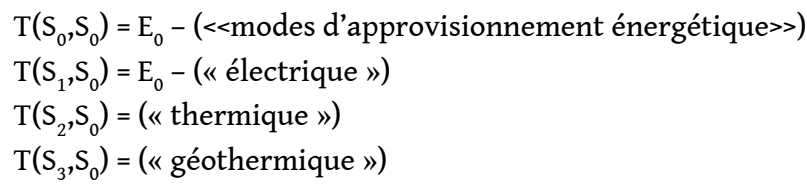

Ici comme dans le précédent exemple, le noyau thématique est introduit en amont des sous-segments régis par les satellites, pour former une structure quasi-énumérative. Le premier satellite apparait sous la forme d'une construction clivée qui, renforcée par un superlatif, annonce l'occurrence d'entités sémantiquement comparables à celle réalisée par $\mathrm{P}_{1}$ (entité dorénavant notée $\mathrm{E}_{1}$ ). C'est effectivement le cas avec $\mathrm{P}_{2}$ et $\mathrm{P}_{3}$, même si la saillance de ces expressions est remarquablement faible. Il est particulièrement intéressant d'observer que la saillance de $\mathrm{P}_{1}$ (vraisemblablement attribuable à la construction clivée), et donc de l'axe sémantique auquel il appartient, semble rendre acceptable l'absence de marque explicite comme « au contraire » ou "de même " pour introduire $\mathrm{S}_{1}$ et $\mathrm{S}_{2}$ : le lecteur initié sera probablement dans l'expectative d'une ou plusieurs entités sémantiquement comparables à $E_{1}$, et leur occurrence effective paraît suffire à faire apparaitre la structure du discours. En d'autres termes, la saillance d'un axe sémantique semble pouvoir participer très activement à l'émergence de la structure discursive.

On voit donc que si le processus de satellisation passe souvent par différentes formes de détachement, cela n'est pas une condition nécessaire. On remarquera toutefois dans cet exemple précis que l'on peut difficilement prêter une fonction réellement 
organisationnelle au syntagme $\mathrm{P}_{2}$, qui n'a aucune portée au delà de la phrase qui l'héberge. On peut cependant observer d'autres configurations discursives très particulières, au sein desquelles ces satellites non détachés semblent bénéficier d'un rôle proprement organisationnel, comme nous allons le voir dans la section suivante.

\subsection{Réalisations discursives sans détachement syntaxique}

"l'enseignement primaire » apparait ici comme sujet de la prédication, et n'est donc plus syntaxiquement détaché. Il est toutefois évident que, tout comme dans l'exemple précédent, $\mathrm{P}_{1}$ fait ici écho à $\mathrm{P}_{2}$, et que l'ensemble du passage reste organisé pour opposer ces deux niveaux du système scolaires. La fonction discursive de $P_{1}$ paraît analogue à celle du premier introducteur de la version précédente, dans la mesure où il spécifie bien un critère d'interprétation s'appliquant au propos central du discours (« le retard scolaire »), et que ce critère vaut pour plusieurs propositions sans être explicitement repris.

Tout se passe donc comme si $\mathrm{P}_{1}$ bénéficiait d'une portée comparable à celle d'un introducteur syntaxiquement détaché, et nous le considérons donc ici comme une forme spécifique d'introducteur d'univers, que nous dirons « intra-prédicatif » (dorénavant $\mathrm{IU}_{\mathrm{IP}}$ ). Notre hypothèse est que nous sommes ici en présence d'une structure discursive fonctionnellement équivalente à la précédente, et que $\mathrm{P}_{1} \mathrm{y}$ joue bien un rôle analogue à celui d'un introducteur. Différents facteurs semblent pouvoir expliquer ce phénomène.

1. i) En premier lieu, il faut considérer avec attention l'antécédent du syntagme pronominal «cette baisse»: il est clair dans ce cas qu'il ne reprend pas seulement le référent de la « forte diminution du retard scolaire », mais bien l'ensemble du contenu propositionnel de l'énoncé qui précède $\left(\mathrm{U}_{1}\right)$, qui pourrait s'exprimer par « la diminution du retard scolaire dans le primaire ». De ce fait, on peut considérer que l'objet sémantique auquel se rapporte la chaîne de référence du segment $S_{1}$ est bien une structure complexe, centrée sur la « forte diminution », mais emportant avec lui « le primaire».

2. ii) En second lieu, la forme même du contenu propositionnel de $U_{1}$ est particulière. En effet, l'acception ici employée du verbe "connaître" correspond ici à un méta-prédicat dont le second argument est lui-même un prédicat. Celui-ci est exprimé par la nominalisation du verbe « diminuer », et son argument est spécifié sous la forme du complément du nom «le taux de retard scolaire ». Or, ce méta-prédicat est relativement neutre, et la structure sémantique résultante peut être "réduite " sans perte d'information en une autre structure dont la formulation la plus immédiate serait «le taux de retard scolaire a diminué dans le primaire ». Vraisemblablement, le choix par le scripteur d'une construction du type « $\mathrm{X}$ a connu $\mathrm{Y}$ » dans un cas comme celui-ci vise la topicalisation de X, qui apparaît ainsi en initiale. Toutefois, du 
fait de son rôle sémantique qui demeure " périphérique ", il semble que cette topicalisation ne suffise pas ici à définir l'à propos de l'énoncé, tout comme un adverbial détaché ne définit pas nécessairement le thème au sens de l'à propos, comme le remarque Charolles dans (Charolles, 2003). Ainsi, dans notre exemple, «l'enseignement primaire » est topicalisé sans pour autant constituer le noyau thématique du segment $S_{1}$, ce qui participe à son accès à la fonction de satellite.

3. iii) Enfin, la portée de $P_{1}$ nous semble également explicable en recourant à la notion d'univers «virtuel» proposée par Charolles (1997). Il se trouve en effet que le syntagme " dans le secondaire ", dont on ne peut douter du statut d'introducteur, projette un univers parent implicite lié à l'ensemble des niveaux scolaires du système éducatif français (pré-élémentaire, primaire, secondaire, supérieur). Or il se trouve que « le primaire » est bien un univers dérivé de cet univers parent, ce qui nous incite probablement à le considérer de façon équivalente à l'univers du «secondaire». Et le fait que l'introducteur au sens strict apparaisse après celui que nous qualifions d'intra-prédicatif ne semble pas problématique si l'on adopte comme M. Charolles une approche incrémentielle, qui prévoit "des possibilités de réanalyse a posteriori avec mise à jour des interprétations construites " (1997 : 3). Nous rejoignons ici la notion d'écho entre ces deux univers, déjà mentionnée plus haut. Les connaissances de domaine semblent ici jouer un rôle important, puisque la familiarité supposée du lecteur avec les niveaux du système scolaire interviennent dans la reconnaissance de la structure discursive du passage comme quasi-énumérative.

41 De nombreux exemples trouvés en corpus relèvent du même phénomène. C'est le cas de l'exemple 6 vu plus haut, ou de l'extrait suivant :

[12] À la fin des années 80, Ullman estimait que ces deux modèles étaient même incompatibles, ce que confirmaient les faits puisque jusqu'alors les bases de données avaient été soit déclaratives mais orientées-valeur, soit orientéesobjet mais non-déclaratives. Cependant, les années 90 ont vu apparaître plusieurs tentatives de conciliations, que nous présentons brièvement dans cette synthèse avant d'en développer deux plus longuement dans le reste de ce chapitre. Ces tentatives peuvent être réparties en deux domaines : les bases de données (monde système) et les bases de connaissances (monde IA). $\mathrm{Au}$ risque d'une simplification excessive, on dira que ces domaines se distinguent par le fait que le premier privilégie les aspects pratiques et l'efficacité, et le deuxième les aspects théoriques et l'expressivité. (Source SIL)

Le suivant se distingue des précédents par le fait qu'il ne recourt pas au procédé du métaprédicat et que l'IU $\mathrm{IP}_{\mathrm{IP}}$ apparaît ici sous la forme d'une extension prépositionnelle dans le syntagme sujet :

[13] § Pour ce qui est du transport ferroviaire, la législation en matière de transport de marchandises dangereuses par rail a été renforcée et, à la suite de la scission de la société nationale des chemins de fer en 5 sociétés au début de 1999, la restructuration du secteur ferroviaire a été poursuivie en $2000[\ldots]$

$\S$ Les activités dans le domaine de la navigation intérieure ont fort souffert du blocage du Danube dû à la crise du Kosovo, ce qui a eu pour conséquence de priver ce secteur des ressources financières nécessaires à son adaptation à l'acquis de l'UE. Les aspects pratiques concernant la conformité des navires 
roumains aux normes de l'UE pourraient poser problème pour des motifs d'ordre économique, eu égard à l'objectif des autorités roumaines d'accès au Rhin. Un décret ministériel a été adopté afin de transposer les règles de l'UE relatives à l'accès à la profession de transporteur de marchandises par voie navigable [...] (Source ROU)

Il est remarquable ici que malgré sa faible saillance au niveau phrastique, le syntagme « le domaine de la navigation intérieure » ait bien une portée significative. Alors que dans les précédents exemples le phénomène de pseudo-détachement semblait jouer un rôle non négligeable dans la perception du rôle des syntagmes concernés, cet $\mathrm{IU}_{\mathrm{IP}}$ apparait ici dans une position "syntaxiquement profonde ", a priori peu encline à lui conférer une portée. Il apparait pourtant que cette portée est bien réelle, puisque si le champ lexical lié à la navigation est significativement présent dans texte qui suit, il n'y a aucune reprise du qualificatif « intérieure », qui est pourtant persistant. D'autre part, il est clair que cet $\mathrm{IU}_{\mathrm{IP}}$ possède bien une fonction d'indexation au même titre que «le transport ferroviaire " auquel il répond. On peut raisonnablement supposer que dans ce cas l'apparition en initiale de paragraphe joue un rôle important, mais là encore la relation sémantique entre plusieurs introducteurs successifs et comparables au sein d'une structure plus globale semble à prendre sérieusement en considération.

Nous formulons l'hypothèse que c'est essentiellement la relation sémantique forte et supposée connue entre ces introducteurs qui autorise l'un (ou même plusieurs) d'entre eux à apparaitre dans une position qui n'est pas explicitement détachée. Et comme nous le verrons dans la section suivante, c'est ce dernier critère qui agira de façon prédominante dans la détection automatique de ces structures discursives particulières.

Plus généralement, nous défendons ici l'hypothèse que des constituants non détachés syntaxiquement peuvent dans certains cas constituer des introducteurs d'univers dotés d'une réelle portée, et spécifier à ce titre des critères d'interprétation portant sur plusieurs propositions sans faire appel aux mécanismes référentiels. Nous avons montré à travers plusieurs exemples que des mécanismes de pseudo-détachement peuvent intervenir (détachement « sémantique » ou « référentiel »), mais aussi que la saillance présupposée de certaines relations sémantiques avec un autre introducteur semble autoriser une absence de marque de détachement explicite.

\section{Analyse automatique de structures en thèmes composites}

\subsection{Principes généraux}

Nous allons maintenant présenter la méthode d'analyse automatique que nous avons élaborée autour de la notion de thème composite. Précisons dès à présent que tous les critères évoqués dans les parties précédentes ne trouvent pas de pendant immédiat dans cette méthode: tous les phénomènes linguistiques susceptibles d'intervenir ne peuvent évidemment pas être automatisés.

47 L'approximation que nous proposons fait interagir deux types d'indices:

- expressions explicitement signalées comme structurantes ;

- expressions considérées comme intrinsèquement structurantes pour le domaine considéré. 

discursifs globaux et de connaissances d'ordre ontologique. Ces dernières sont formalisées sous forme d'axes sémantiques, qui correspondent à des espaces notionnels susceptibles de participer à l'indexation de l'information dans les textes considérés. Il pourra s'agir d'axes génériques comme le temps ou espace, ou d'axes plus spécifiques à un domaine ou à une pratique (axe des niveaux scolaires, des types de transports, etc.).

\subsection{Méthode}

51 Le procédé d'analyse ici décrit débute par une segmentation fondée sur l'analyse des satellites, avant de procéder à l'identification des noyaux au sein des segments obtenus. La première phase du traitement consiste à baliser les unités lexicales ou syntagmatiques susceptibles de jouer un rôle dans l'organisation du discours, sous la forme de connecteurs discursifs ou de termes apparaissant dans les axes sémantiques connus du système. Ces derniers sont donnés sous la forme de listes d'entrées lexicales ou de grammaires syntagmatiques (dans le cas du temps ou de l'espace par exemple). La segmentation proprement dite est fondée sur un catalogue de configurations discursives 
prédéterminées, ces dernières pouvant être réparties en trois catégories selon la nature des critères qui les déterminent :

1. i) Les configurations explicites décrivent des structures intégralement marquées par des indices accessibles par leur forme de surface. Il s'agit notamment de différentes formes de détachement syntaxique et notamment d'introducteurs de cadre (si la question de leur portée est très complexe, ces expressions sont généralement facilement détectables).

2. ii) Les configurations mixtes concernent les situations où la structure n'est pas intégralement marquée, et dont l'analyse requiert un recours aux connaissances du domaine, comme dans la plupart des exemples présentés dans la section précédente.

3. iii) Enfin, les configurations implicites regroupent les cas où aucune marque discursive n'a pu être repérée. Ces passages pourront être segmentés si différents éléments d'un même axe sémantique y apparaissent simultanément alors qu'aucune autre configuration n'a pu être reconnue.

52 Notons dès à présent que cette typologie ne traduit pas l'existence de procédés d'analyse fondamentalement différents pour chacune des catégories, mais permet d'établir des règles de priorité permettant de traiter les conflits entre différentes alternatives, sur lesquelles nous reviendrons ci-après.

53 Notre méthode de reconnaissance automatique de ces configurations présuppose la définition d'une unité textuelle maximale au sein de laquelle elles seront recherchées. Cette unité est pour l'heure définie comme correspondant au paragraphe, ce qui recouvre une partie conséquente des structures concernées dans notre corpus, mais devra bien sûr être généralisé à l'avenir. Conformément aux principes évoqués plus haut, le procédé débute par la recherche d'expressions apparaissant dans une position discursive caractéristique (introducteur, clivée, connecteur discursif, etc.) et/ou appartenant à un axe sémantique connu. Dans un second temps, les expressions ainsi repérées et appartenant à un même axe sémantique sont mises en relation, chaque ensemble obtenu constituant alors une instance en discours de l'axe associé. À ce stade, chacune de ces instances est une source potentielle de segmentation.

Dans beaucoup de cas, un même segment présentera simultanément plusieurs instances d'axes et donc plusieurs segmentations potentielles. Pour résoudre ces conflits, nous utilisons une heuristique visant à préférer systématiquement les configurations explicites aux configurations mixtes, qui seront elles-mêmes préférées aux configurations implicites. L'hypothèse sous-jacente est que la signalisation explicite de la structure du discours prendra le pas sur des structures dont l'interprétation nécessite diverses inférences. De la même manière, dans le cas où plusieurs configurations mixtes se présentaient simultanément, celle qui présentera le plus grand nombre de satellites explicitement signalés sera préférée. Il va de soi que cette heuristique devra à l'avenir être évaluée de façon systématique, et très probablement complétée par des règles portant sur d'autres critères.

La structure que nous recherchons étant par nature hiérarchique, le processus d'analyse doit inclure une forme de récursivité permettant de détecter les configurations enchâssées. Pour l'heure, le système considère tout d'abord le grain maximal évoqué plus haut, avant d'appliquer le même processus aux segments obtenus lors de cette première passe. Cela sera répété jusqu'à une profondeur préalablement choisie ou jusqu'à ce qu'aucune configuration ne soit plus reconnue. 
ne fois la segmentation obtenue, le système procède à l'identification des noyaux pour chaque segment afin d'obtenir une représentation complète de leur thème composite. Ceux-ci sont obtenus par une méthode quantitative permettant d'obtenir pour chaque segment une liste de syntagmes distributionnellement saillants. Cet aspect combine ainsi la modélisation linguistique et une méthode numérique, mais il serait possible d'employer un procédé différent, qui pourrait par exemple se fonder sur une analyse plus fine de la structure informationnelle. Notons que si notre représentation des thèmes composites n'admet en principe qu'un seul noyau pour chaque segment, il semble intéressant dans la perspective de la recherche d'information de conserver plusieurs noyaux potentiels pour chaque segment. En effet, il ne s'agit pas tant d'obtenir une représentation idéale sur le plan linguistique que de produire une indexation susceptible de donner les meilleurs résultats possibles à l'utilisateur. Dans cette application, nous représentons donc chaque noyau par une liste de termes triée par ordre de pertinence.

\subsection{Implémentation}

Le procédé est implémenté sous LinguaStream ${ }^{3}$ (Bilhaut, Widlöcher, 2006), plate-forme permettant d'intégrer les différents modules d'analyse sous forme de chaînes de traitement. Ces chaînes peuvent être conçues visuellement au sein de l'environnement graphique de la plate-forme, en assemblant des "briques" correspondant à chaque traitement et en exprimant des règles à l'aide de différents formalismes déclaratifs. La chaîne implémentant la méthode présentée plus haut est reproduite par la figure 1, telle qu'elle apparait dans l'interface de la plate-forme. Chaque rectangle correspond à une étape du traitement, les flux de données (texte et annotations) étant figurés par les flèches.

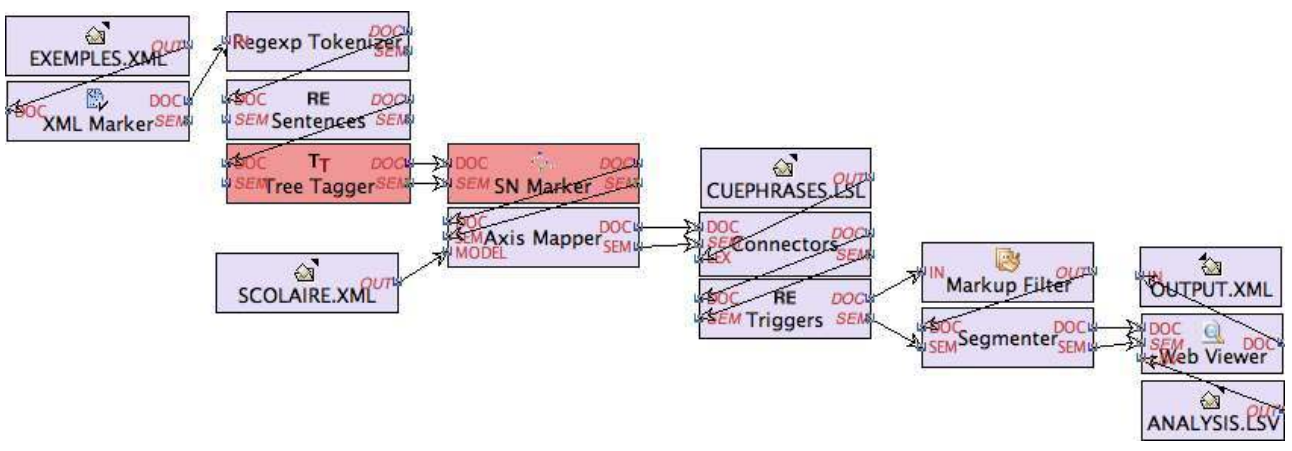

Figure 1 : Chaîne de traitement de l'analyseur de thèmes composites

À la suite des composants classiques qui procèdent aux découpages initiaux (mots, phrases) et à l'analyse morpho-syntaxique, le composant "SN Marker» se charge d'identifier les syntagmes nominaux à l'aide d'une grammaire locale d'unification. Par la suite, le composant «Axis Mapper » se charge de reconnaître les termes appartenant à un axe sémantique connu, étant donné un ensemble d'axes constituant les ressources propres au domaine (ici dans le fichier « scolaire.xml »). On notera que ce composant ne se charge que des axes qui sont spécifiés par des listes d'entrées lexicales. Dans le cas où une analyse spécifique est nécessaire (comme pour le temps ou l'espace, qui constituent des axes pertinents dans de nombreux cas), il est nécessaire d'insérer dans la chaîne des composants adaptés (non reproduits ici). 

(composant "Connectors »), et un ensemble de patrons donnés sous forme de règles séquentielles permettent de reconnaître les termes apparaissant dans une position caractéristique de la fonction satellite (introducteurs, clivées, initiale de paragraphe, etc.). À ce stade, sont donc marqués dans le texte d'une part les termes appartenant à un axe sémantique connu, et d'autre part ceux dont la fonction est marquée à la surface du texte.

La dernière phase du traitement, réalisée par le composant "Segmenter » procède à la segmentation proprement dite, en cherchant à reconnaître les instances des configurations évoquées dans la section précédente avant d'appliquer les règles de priorité. Il s'agit pour l'heure d'un composant ad-hoc, chaque configuration particulière étant «implémentée " par une classe Java spécifique. De façon à exprimer ces configurations par des règles déclaratives, nous travaillons actuellement à mettre à l'oeuvre le langage $\mathrm{CDML}^{4}$ (Widlöcher, 2006), qui permettra dans une version ultérieure d'exprimer sous forme de "grammaire de discours " l'ensemble des configurations qui nous intéressent ici. Nous nous baserons alors sur les mêmes traitements en amont, seul le composant « Segmenter » étant à remplacer.

61 On notera que nous ne traitons ici que très partiellement le problème de la portée des expressions satellites, intégrant uniquement le procédé d'analyse de cadres temporel décrit dans (Bilhaut et al., 2003). Dans tous les autres cas (il n'existe pas à l'heure actuelle de méthode générique pour évaluer ces portées), le système considère que la borne finale du dernier segment de chaque configuration se termine nécessairement avec le segment englobant. On remarquera également que la chaîne ici reproduite ne gère pas par ellemême la récursivité des structures à analyser. En effet, il est pour l'heure nécessaire de répéter le composant "Segmenter» pour obtenir une structure hiérarchique: la première passe s'attachera à un grain maximal fixé à l'avance (typiquement le paragraphe ou la section), alors que les suivantes analyseront les segments établis lors des passes précédentes.

\begin{tabular}{l}
\hline Le retard scolaire dans les années 80 \\
$\S$ Les taux d'échec scolaire ont sensiblement varié \\
durant cette période. \\
$\begin{array}{l}\text { Dans le privé, en particulier, on a pu observer une } \\
\text { hausse significative du nombre de [...]. }\end{array}$ \\
$\begin{array}{l}\text { Le secteur public n'échappe pas à la règle, même } \\
\text { si les motifs de redoublement diffèrent [...]. }\end{array}$ \\
$\begin{array}{l}\text { C'est dans l'est de la Bretagne que ce } \\
\text { phénomène est le plus sensible, probablement } \\
\text { en raison de [...]. }\end{array}$ \\
\hline
\end{tabular}

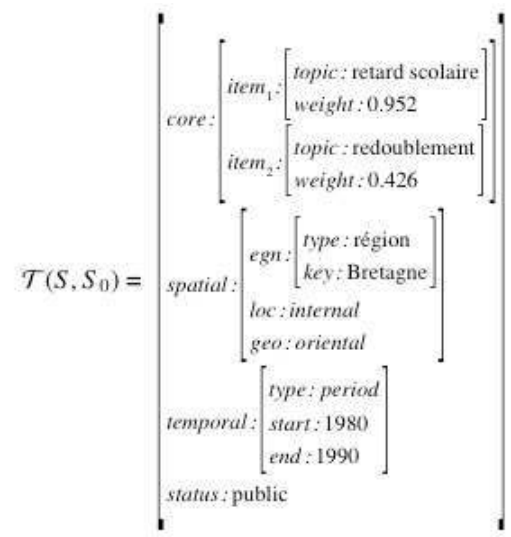

$$
\left\{\begin{array}{l}
\mathcal{T}\left(S_{0}, S_{0}\right)=\text { RETARD SCOLAIRE } \bullet([1980 ; 1990],\langle\langle\text { STATUT }\rangle\rangle) \\
\mathcal{T}\left(S, S_{0}\right)=\text { RETARD SCOLAIRE } \bullet \bullet([1980 ; 1990], \text { PUBLIC, BRETAGNE ORIENTALE })
\end{array}\right.
$$

Figure 2 : Résultat d'une analyse automatique en thèmes composites. À droite le résultat de l'analyse automatique proprement dite, sous forme d'une structure de traits, et en dessous la représentation symbolique équivalente. 
62 résultats qualitativement intéressants qui devront par la suite faire l'objet d'une
évaluation quantitative. Un exemple de résultat est présenté en figure 2, où apparaît une
structure arborescente centrée sur le noyau thématique « retard scolaire », et articulée
autour des axes du temps, du statut (public ou privé) des établissements scolaires, et de
l'espace. Ces données sont directement applicables dans le contexte de l'accès assisté à
l'information, le système produisant à cette fin un index intra-documentaire constitué
d'une représentation XML des structures de traits représentant les thèmes, index
exploitable par un moteur de recherche spécifique (Bilhaut et al., 2007).

\section{Conclusion et perspectives}

63

Nous avons détaillé dans cet article une approche de l'analyse thématique automatique dont les spécificités sont la formalisation et la détection de structures discursives spécifiquement liées à un phénomène sémantique bien défini, ainsi que l'intervention dans le procédé d'analyse de ressources d'ordre ontologique liées au domaine. Nous avons également envisagé les corollaires de cette approche en termes de représentation des connaissances et d'acquisition automatique des ressources nécessaires.

Si la mise en œuvre automatique de la démarche est un problème très complexe et pourra être largement améliorée à l'avenir, les premières expérimentations que nous avons réalisées sont très encourageantes et pourront à court terme être évaluées en contexte, au sein d'un système de recherche documentaire que nous avons développé à cette fin. L'application la plus immédiate est un moteur de recherche multicritères spécifiquement adapté à un domaine donné : l'utilisateur peut y spécifier le thème principal de sa requête (qui correspondra dans les documents retournés à une entité noyau) ainsi qu'un certain nombre de critères supplémentaires qui correspondront dans les documents à des entités satellites. Un exemple de requête dans le domaine géographique pourrait être: «vieillissement de la population ; dans l'Ouest ; depuis les années 90 ». D'autres applications exploitant plus directement la segmentation du texte pour aider à la navigation intra-documentaire pourront être envisagées par la suite. Au delà de l'évaluation qualitative et extrinsèque que nous espérons retirer de ces expérimentations, une évaluation intrinsèque doit également être envisagée, mais demande un important travail préliminaire à la fois en termes méthodologiques et d'annotation.

Concernant le modèle lui-même, il sera également nécessaire de recenser d'autres configurations qui, comme nous avons pu l'observer sur différents exemples, semblent faire intervenir des phénomènes de portée sans pour autant impliquer d'introducteur au sens strict, ni même, dans certains cas, de constructions syntaxiquement détachées. Nous avons notamment évoqué à ce sujet la possibilité de rencontrer des phénomènes de détachement qui seraient d'une autre nature (notamment "référentielle» ou « sémantique »), que nous souhaitons explorer plus avant. Nous envisageons par ailleurs de considérer d'éventuels points de jonction entre la notion de portée en général et des structures d'ordre rhétorique.

Une autre voie consisterait à explorer les mécanismes analogues mais qui concernent des niveaux de grain différents. Leur analyse pourrait venir compléter celle des structures discursives qui ont retenu notre attention jusqu'ici, et il s'agirait donc d'envisager en parallèle différentes manifestations d'un même phénomène thématique, de façon à 
obtenir, toujours sous l'angle particulier des thèmes composites, une couverture textuelle plus importante. Cela concerne d'une part des objets de grain plus fin, touchant notamment au plan terminologique. On voit bien effet qu'un thème composite tel que « le retard scolaire » (« dans le secondaire ») pourra aussi bien être instauré par un syntagme tel que « le retard scolaire dans le secondaire » que par une structure cadrative du type «Dans le secondaire, le retard scolaire...", même si les mécanismes assurant leur persistance en discours seront différents. Cela concerne d'autre part des structures de grain élevé, et notamment celles de l'architecture textuelle. Il est en effet fréquent, dans les textes expositifs en particulier, que des thèmes composites soient instaurés par des successions de titres.

Une dernière piste, transversale à celles que nous venons d'évoquer, concerne l'identification des noyaux thématiques au sein des structures en thèmes composites. Nous avons vu que notre méthode d'analyse automatique est fortement centrée sur les satellites thématiques, et que nous nous sommes rangés à des méthodes relativement classiques pour ce qui est des noyaux. Même si cela a été l'occasion de mettre en pratique la collaboration entre méthodes "linguistiques » et "quantitatives ", nous souhaitons également envisager la possibilité d'exploiter des concepts gravitant autour des notions de structure informationnelle, de chaîne de référence, et de progression thématique. Nous rejoindrions en cela la problématique de l'interaction entre portée et continuité référentielle.

\section{BIBLIOGRAPHIE}

BILHAUT, F., HO-DAC, L.-M., BORILlO, A., CHARNOIS, T., ENJALBERT, P., LE DRAOULEC, A., MATHET, Y., MIGUET, H., PÉRY-WOODLEY, M.-P., SARDA, L. 2003. Indexation discursive pour la navigation intradocumentaire : cadres temporels et spatiaux dans l'information géographique. Actes de la 10e Conférence Traitement Automatique du Langage Naturel (TALN'03). Batz-sur-Mer, France. 315-320.

BILHAUT, F., WIDLÖCHER, A. 2006. LinguaStream: An Integrated Environment for Computational Linguistics Experimentation. Proceedings of the 11th Conference of the European Chapter of the Association of Computational Linguistics (EACL'06). Trento, Italie. 95-98.

BILHAUT, F., DUMONCEL, F., ENJALBERT, P., HERNANDEZ, N. 2007. Indexation sémantique et recherche d'information interactive. Actes de CORIA 2007, Quatrième conférence francophone en Recherche d'Information et Applications. Saint-Etienne, France. 65-76.

CHAFE, W. L. 1976. Givenness, Contrastiveness, Definiteness, Subjects, Topics, and Point of View. Subject and Topic, Li C. N. (ed.). New York: Academic Press. 25-55.

CHAROLLES, M. 1997. L'encadrement du discours - Univers, champs, domaines et espaces. Cahiers de recherche linguistique $6: 1-73$.

CHAROlles, M. 2003. De la topicalité des adverbiaux détachés en tête de phrase. Travaux de linguistique : Adverbiaux et topiques 47. M. Charolles, S. Prévost (éd). Gent : Rijksuniversiteit van Gent. 11-51. 
DIK, S. C. 1989. The Theory of Functional Grammar. Dordrecht: Foris Publications.

FERRET, O., GRAU, B. 2001. Utiliser des corpus pour amorcer une analyse thématique. Traitement Automatique des Langues 42 (2) : 517-545.

HERNANDEZ, N. 2004. Description et détection automatique de structures de texte. Thèse de doctorat. Paris : Université Paris Sud-Orsay.

LAMBRECHT, K. 1994. Information Structure and Sentence Form: Topic, Focus and the Mental Representation of Discourse Referents. Cambridge : Cambridge University Press.

LE GOFFIC, P. 1994. Grammaire de la phrase française. Paris : Hachette.

LOWE, I. 1987. Sentence initial elements in english and their discourse function. Occasional Papers in Systemic Linguistics 2: 5-34.

LUCAS, N., GIGUET, E. 2005. UniTHEM, un exemple de traitement linguistique à couverture multilingue. Actes de la 8e Conférence Internationale sur le Document Electronique (CIDE.8). Beyrouth, Liban. 115-132.

MARTIN, R. 1983. La logique du sens. Paris : Presses Universitaires de France.

VAN DIJK, T. A. 1977. Text and Context. London : Longman.

WIDLÖCHER, A. 2006. Analyse par contraintes de l'organisation du discours. Actes de la 13e conférence sur le Traitement Automatique des Langues Naturelles (TALN 2006). Louvain-laNeuve : Presses universitaires de Louvain. 367-376.

\section{ANNEXES}

Sources des extraits de corpus

APH : Oria, M., Raffin, J. 1973. Anatomie, Physiologie, Hygiène. Paris : Hatier.

BEA : Bulletins REC Info du Bureau d'Enquête et Analyses (BEA) pour la sécurité de l'aviation civile. http://www.bea-fr.org

EC : Étude de «Énergie-Cités », association des autorités locales européennes pour une politique énergétique locale durable. Rapport sur « Lüchow-Dannenberg ». http:// www.energie-cites.org

HER : R. Hérin, R. Rouault, V. Veshambre, 1994. Atlas de la France scolaire de la maternelle au lycée. Collection « Dynamiques du territoire ». Reclus.

ROU : Synthèse d'un rapport de la communauté européenne sur les transports en Roumanie.

SIL : S. Ferré, 2002. Systèmes d'information logiques, un paradigme logico-contextuel pour interroger, naviguer et apprendre. Thèse de doctorat. Rennes : Université Rennes 1.

\section{NOTES}

1. Nous verrons plus loin que ces deux approches ne doivent cependant pas être considérées comme incompatibles.

2. Notons que cela ne nous conduira pas nécessairement à considérer que l'un ou l'autre est plus « valide » que les autres, puisque dans notre perspective applicative on pourra effectivement être amené à considérer un segment à l'exclusion d'une part de son contexte. 


\section{RÉSUMÉS}

Le travail présenté dans cet article s'inscrit dans la problématique de l'analyse thématique automatique du discours. Nous proposons un modèle de représentation des thèmes textuels fondé sur la notion d'univers de discours, que nous appliquons à travers différents exemples à diverses configurations discursives. Nous illustrons le fait que des connaissances relatives à un domaine de spécialité peuvent intervenir dans l'organisation du discours qui s'y rapporte. Nous présentons enfin une méthode d'analyse automatique du discours basée sur ce modèle, applicable au domaine de l'accès assisté à l'information.

This paper focuses on the issue of automatic thematic analysis at the discourse level. We propose a representational model of textual topics based on the notion of "discourse universe", applied to various discursive patterns through several examples. We illustrate the fact that domain-specific knowledge can play a significant role in the structure of the related discourse. We finally introduce an automatic analysis method founded on this model and applicable to information retrieval tasks.

\section{INDEX}

Keywords : discourse, semantics, discourse universe, thematic analysis

Mots-clés : discours, sémantique, univers de discours, analyse thématique

\section{AUTEUR}

\section{FRÉDÉRIK BILHAUT}

GREYC - Université de Caen - CNRS UMR 6072 